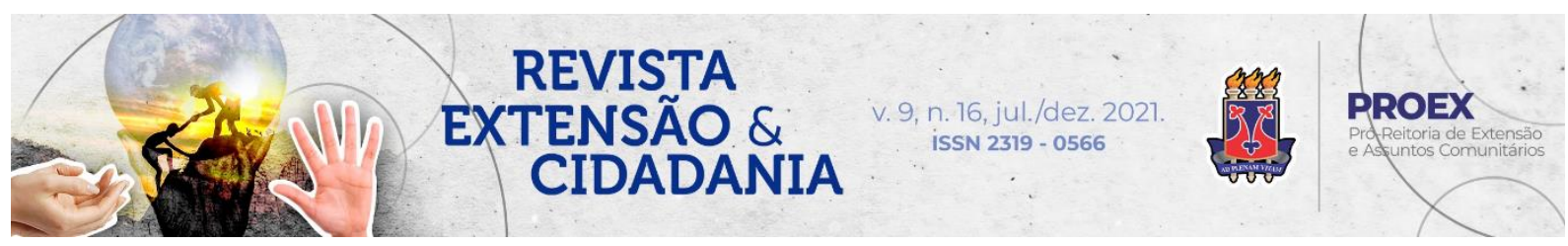

DOI: $10.22481 /$ recuesb.v9i16.9110

\title{
DESVENDANDO A ANATOMIA CARDÍACA POR MEIO DA DISSECAÇÃO: RELATO DE EXPERIÊNCIA DA LADHAS UNIFAL-MG
}

\author{
UNVEILING THE CARDIAC ANATOMY THROUGH DISSECTION: AN \\ EXPERIENCE REPORT FROM LADHAS UNIFAL-MG
}

\section{DEVELANDO LA ANATOMÍA CARDIACA A TRAVÉS DE LA DISECCIÓN: RELATO DE EXPERIENCIA DE LADHAS UNIFAL-MG}

\author{
Gabriel dos Reis Pinto ${ }^{1}$ \\ Paula Camelo de Almeida Santos ${ }^{2}$ \\ Barbara Bianca Melo Toledo 3 \\ Gabriel Ferro Baccaro ${ }^{4}$ \\ Isadora Furlan Ribeiro ${ }^{5}$ \\ Evelise Aline Soares ${ }^{6}$
}

Resumo: O estudo da anatomia do sistema cardiovascular colabora para o entendimento da fisiopatologia da hipertensão arterial sistêmica, doença de elevada prevalência mundial, sendo ela um dos focos das atividades de extensão da Liga Acadêmica de Diabetes e Hipertensão Arterial Sistêmica do curso de Medicina da Universidade Federal de Alfenas. Buscando uma integração básico-clínica, por meio de metodologias ativas, e estimulando as habilidades de comunicação dos extensionistas da liga, foi criado um evento com o intuito de promover uma revisão do sistema cardiovascular. Tendo a dissecação do coração como principal elemento atrativo, o evento proporcionou aos ingressantes do curso de Medicina o primeiro contato com os instrumentais cirúrgicos, as normas de biossegurança e a dissecação de vísceras frescas. $\mathrm{O}$ presente artigo tem por objetivo relatar a experiência sob a perspectiva dos extensionistas e dos participantes do evento "Anatomia cardíaca: dissecação do coração", analisando o impacto

\footnotetext{
1 Graduando em Medicina, Universidade Federal de Alfenas, Alfenas, Minas Gerais, Brasil. Orcid: https://orcid.org/0000-0002-4794-1110Ｅ-mail: gabriel.dosreis@sou.unifal-mg.edu.br

2 Graduanda em Medicina, Universidade Federal de Alfenas, Alfenas, Minas Gerais, Brasil. Orcid: https://orcid.org/0000-0003-4881-9026 E-mail: paula.santos@ sou.unifal-mg.edu.br

3 Graduanda em Medicina, Universidade Federal de Alfenas, Alfenas, Minas Gerais, Brasil. Orcid: https://orcid.org/0000-0003-1986-8072 E-mail: barbara.toledo@sou.unifal-mg.edu.br

4 Graduando em Medicina, Universidade Federal de Alfenas, Alfenas, Minas Gerais, Brasil. Orcid: https://orcid.org/0000-0001-7467-1657 E-mail: gabriel.baccaro@ sou.unifal-mg.edu.br

5 Graduanda em Medicina, Universidade Federal de Alfenas, Alfenas, Minas Gerais, Brasil. Orcid: https://orcid.org/0000-0002-9421-0505 E-mail: isadora.ribeiro@sou.unifal-mg.edu.br

${ }^{6}$ Fonoaudióloga; Doutora na área de Anatomia, pelo programa de Biologia Celular e Estrutural da Universidade Estadual de Campinas. Professora da Faculdade de Medicina, da Universidade Federal de Alfenas, Alfenas, Minas Gerais, Brasil. Orcid: http://orcid.org/0000-0001-7838-687X E-mail: evelise.anatomia@gmail.com
} 
deste na formação dos estudantes envolvidos. Participaram da atividade 120 estudantes do curso de Medicina da UNIFAL-MG. Foram utilizados kits de instrumentais cirúrgicos, luvas, coração suíno, jaleco e o roteiro que continha as etapas da dissecação. Dessa maneira, a experiência do evento para os extensionistas foi de extrema importância para o desenvolvimento de habilidades comunicativas, da aprendizagem a partir do ensinamento e do trabalho em equipe. Já para os participantes, a atividade permitiu um melhor conhecimento da anatomia cardíaca e o primeiro contato com os instrumentais cirúrgicos.

Palavras-chave: Coração. Dissecação. Hipertensão arterial.

Abstract: The cardiovascular system anatomy study contributes to understanding the pathophysiology of systemic arterial hypertension, a high worldwide prevalence disease and one of the extension activities focuses of the Academic League of Diabetes and Systemic Arterial Hypertension of the Federal University of Alfenas. Searching a basic-clinical integration through the active methodologies and stimulating the league extensionists' communication skills, it was created an event in order to promote a cardiovascular system review. Being the heart dissection the main attraction, the event provided to the medical students a first contact with surgical instruments, biosafety standards and fresh viscera dissection. This article aims to report the experience of the event "Cardiac anatomy: dissection of the heart" from the perspective of its league extensionists and participants, analyzing the impact on their education. 120 students from the medical course at UNIFAL-MG participated in the activity. Dissection kits, gloves, swine hearts, lab coats and the dissection script containing step-by-step dissection were used. In this way, the event experience for the league extensionists was extremely important for developing communication skills, learning by teaching and teamwork. For the participants, the activity improved their knowledge about cardiac anatomy and allowed the first contact with surgical instruments.

Keywords: Heart. Dissection. Hypertension.

Resumen: El estudio de la anatomía del sistema cardiovascular contribuye al conocimiento de la fisiopatología de la hipertensión arterial sistémica, enfermedad de alta prevalencia mundial, siendo ella uno de los focos de las actividades de divulgación de la Liga Académica de Diabetes e Hipertensión Arterial Sistémica de la Facultad de Medicina de la Universidad Federal de Alfenas. Buscando una integración básica-clínica, a través de metodologías activas y estimulando las habilidades comunicativas de los extensionistas de la liga, se creó un evento con el fin de promover una revisión del sistema cardiovascular. Con la disección del corazón como principal elemento atractivo, el evento brindó a los integrantes de la carrera de Medicina el primer contacto con el instrumental quirúrgico, los estándares de bioseguridad y la disección de vísceras frescas. Este artículo tiene como objetivo relatar la experiencia desde la perspectiva de los extensionistas y participantes del evento "Anatomía cardíaca: disección del corazón", analizando su impacto en la formación de los estudiantes involucrados. En la actividad participaron 120 estudiantes de la carrera de Medicina de UNIFAL-MG. Se utilizaron kits con instrumentales quirúrgicos, guantes, corazón de cerdo, bata de laboratorio y el guión que contenía los pasos de la disección. Así, la experiencia del evento para los extensionistas fue de suma importancia para el desarrollo de las habilidades comunicativas, del aprendizaje a partir de la enseñanza y el trabajo en equipo. En cuanto a los participantes, la actividad permitió un mejor conocimiento de la anatomía cardíaca y el primer contacto con los instrumentales quirúrgicos.

Palabras clave: Corazón. Disección. Hipertensión.

Revista Extensão \& Cidadania, v. 9, n. 16, p. 153-165, jul./dez. 2021.

ISSN 2319-0566

DOI: $10.22481 /$ recuesb.v9i16.9110 


\section{Introdução}

A dissecação é um método pedagógico utilizado no estudo da anatomia que permite a observação dos detalhes das estruturas e da presença de variações morfológicas, contribuindo de forma significativa para a construção do aprendizado (PONTINHA; SOEIRO, 2014). Entretanto, devido à dificuldade de acesso a peças cadavéricas humanas (COLARES et al., 2019), a utilização de órgãos de mamíferos, como suínos e equinos, torna-se uma opção para o ensino-aprendizado por meio da anatomia comparada, já que algumas estruturas desses animais, como o coração, possuem semelhanças com os órgãos humanos (LEMOS, 2017).

O conhecimento da anatomia humana é fundamental para a formação médica, o estudo da anatomia do sistema cardiovascular colabora para o entendimento da fisiopatologia da Hipertensão Arterial Sistêmica (HAS). A HAS é uma condição clínica definida como o aumento sustentado da pressão arterial sistólica a valores acima de $140 \mathrm{mmHg}$ e/ou da pressão arterial diastólica a valores acima de $90 \mathrm{mmHg}$ (BARROSO et al., 2021), sendo que 32,3\% dos brasileiros possuem uma pressão arterial compatível com o diagnóstico de HAS e/ou utilizam algum medicamento anti-hipertensivo (MALTA et al., 2016).

Frente a isso, buscando integrar ações de ensino, pesquisa e extensão sobre a HAS, assim como sobre o diabetes, em 12 de dezembro de 2017, foi criada a Liga Acadêmica de Diabetes e Hipertensão Arterial Sistêmica (LADHAS), do curso de Medicina, da Universidade Federal de Alfenas (UNIFAL-MG).

A LADHAS é supervisionada pela coordenadoria de extensão e ligas médicas do centro acadêmico e pela Pró-Reitoria de Extensão da universidade. Além disso, a liga é coordenada por duas professoras da Faculdade de Medicina da UNIFAL-MG, sendo uma delas anatomista e a outra médica de família e comunidade. A proposta da LADHAS é, junto aos extensionistas, desenvolver ações relacionadas ao diabetes e à HAS, priorizando a interdisciplinaridade e promovendo a saúde de modo democrático à população geral e à comunidade acadêmica.

Buscando uma integração básico-clínica, com o foco na HAS, utilizando-se de metodologias ativas e estimulando as habilidades de comunicação dos extensionistas da Liga, foi criado um evento com o intuito de promover uma revisão do sistema cardiovascular. Tendo a dissecação do coração como principal elemento atrativo, o evento proporcionou aos

Revista Extensão \& Cidadania, v. 9, n. 16, p. 153-165, jul./dez. 2021. 
ingressantes do curso de Medicina o primeiro contato com os instrumentais cirúrgicos, as normas de biossegurança e a dissecação de vísceras frescas.

Diante do exposto, o presente artigo tem por objetivo relatar a experiência sob a perspectiva dos extensionistas e dos participantes do evento "Anatomia cardíaca: dissecação do coração", analisando seu impacto na formação dos estudantes envolvidos.

\section{Metodologia}

Trata-se de um relato de experiência a respeito de uma ação de extensão realizada no âmbito da LADHAS, do curso de Medicina, da UNIFAL-MG. A atividade da Liga foi realizada no Laboratório 01, do Departamento de Anatomia. A ação foi registrada no Controle de Ações de Extensão da Universidade com o nome "Anatomia cardíaca: dissecação do coração", na qual os estudantes do primeiro ano do curso de Medicina da UNIFAL-MG puderam realizar a inscrição, assim como estudantes de outra instituição de ensino superior de Alfenas.

A atividade ocorreu duas vezes até então, sendo a primeira em 2018 e a segunda em 2019. Participaram da atividade 60 estudantes ingressantes no ano de 2018 e outros 60 ingressantes no ano de 2019, totalizando, nas duas edições do evento, 120 participantes matriculados no primeiro ano do curso médico. Na realização de cada evento, a turma $(n=60)$ foi dividida em três subturmas $(n=20)$, facilitando o monitoramento dos estudantes durante a parte prática de dissecação, repetindo o evento por três dias consecutivos.

\section{Etapas do desenvolvimento da atividade}

Na primeira etapa, após a inscrição dos participantes, os extensionistas da LADHAS encaminharam um e-mail aos inscritos contendo as instruções gerais sobre materiais para uso durante o evento, conforme descrito no Quadro 1.

Revista Extensão \& Cidadania, v. 9, n. 16, p. 153-165, jul./dez. 2021. 


\section{Quadro 1 - Instruções enviadas aos inscritos}

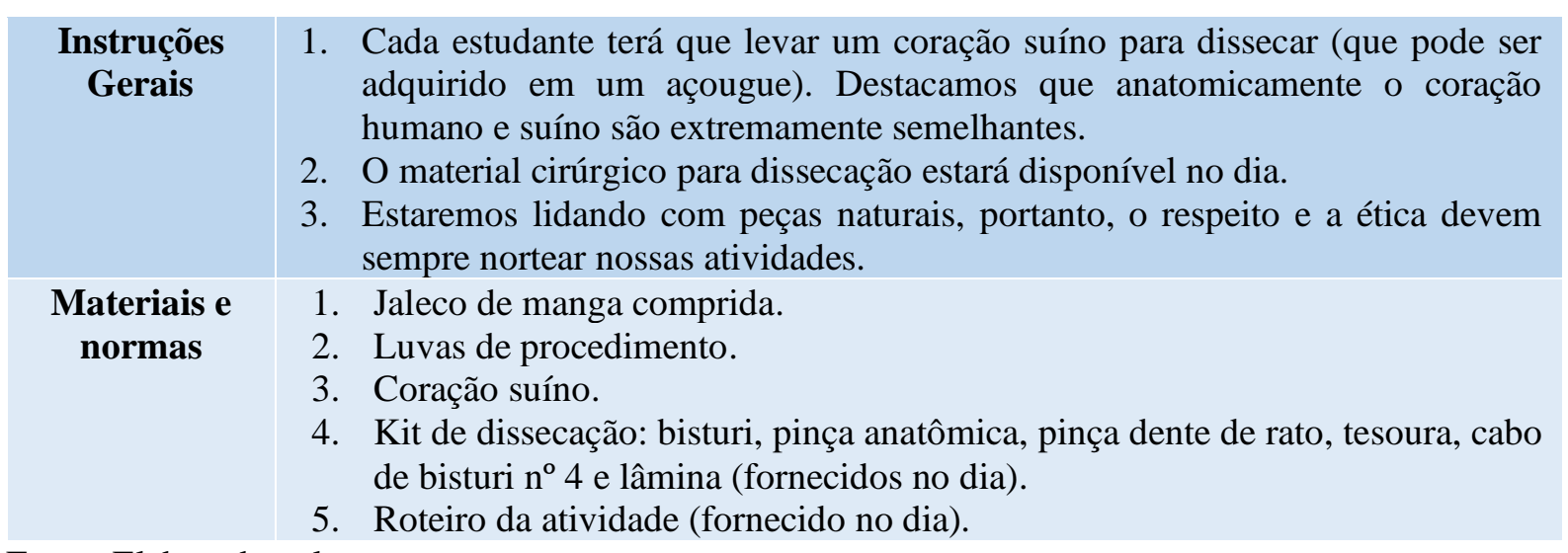

Fonte: Elaborado pelos autores.

Na segunda etapa, os extensionistas da LADHAS elaboraram uma revisão teórica sobre a anatomia do coração e dos grandes vasos da base, norteada pela bibliografia recomendada no plano de ensino da disciplina de Anatomia Aplicada à Medicina. O material teórico foi criado em apresentação de slide, sendo supervisionado pela docente responsável pela disciplina, que também é uma das coordenadoras da Liga Acadêmica. Para viabilizar a apresentação, os extensionistas utilizaram um projetor multimídia e a apresentação teórica durou aproximadamente 40 minutos, sendo aberto, posteriormente, um espaço para as perguntas dos participantes do evento.

$\mathrm{Na}$ terceira etapa, os extensionistas distribuíram os estudantes no laboratório de modo que, em cada bancada, ficassem cinco participantes e um extensionista, sendo este responsável pela supervisão da atividade prática de sua bancada. Após a distribuição dos estudantes, foi realizada uma apresentação dos materiais cirúrgicos pertencentes aos kits de dissecação (tesoura, pinça anatômica, pinça dente de rato, cabo de bisturi n. ${ }^{\circ} 4$ e lâminas de bisturi), além dos cuidados relacionados à biossegurança e ao uso correto dos instrumentais durante a atividade de dissecação (MIZERES; GARDNER, 1988; WEBER, 2001; RODRIGUES, 2010). Em seguida, os estudantes foram orientados sobre a sequência de dissecação.

$\mathrm{Na}$ quarta etapa, o roteiro de dissecação foi elaborado pelos membros da LADHAS, conforme apresentado no Quadro 2.

Revista Extensão \& Cidadania, v. 9, n. 16, p. 153-165, jul./dez. 2021. 


\section{Quadro 2 - Roteiro para dissecação do coração}

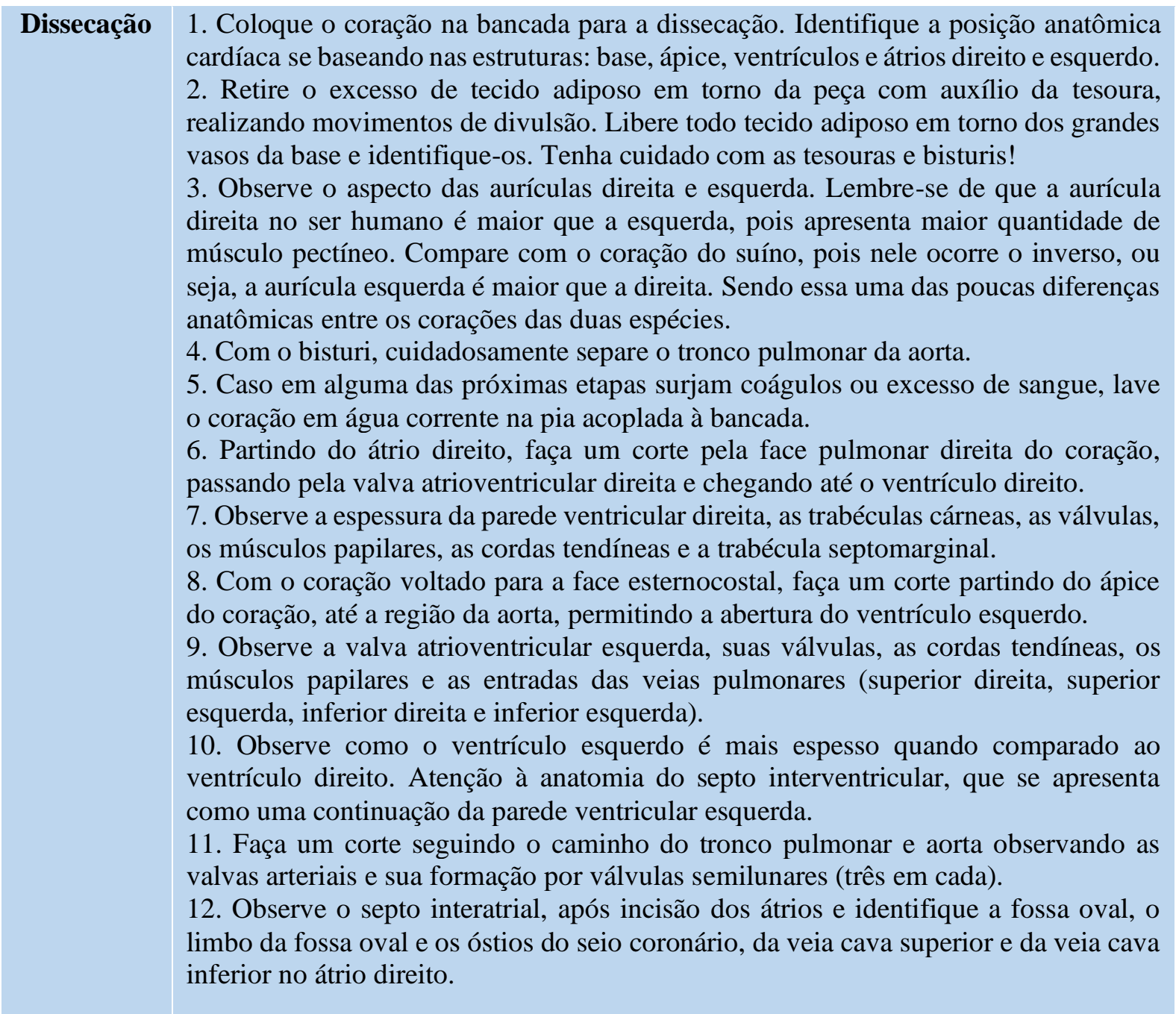

Fonte: Elaborado pelos autores.

$\mathrm{Na}$ quinta etapa, após a realização das dissecações, os estudantes organizaram o laboratório e descartaram os materiais residuais, as lâminas de bisturi foram corretamente descartadas no lixo reservado para materiais perfurocortantes. Os corações suínos utilizados na atividade foram recolhidos e entregues ao técnico do laboratório para que realizasse o descarte correto do material. Por fim, os membros da LADHAS apresentaram um resumo sobre as atividades desenvolvidas pela Liga, a importância da anatomia cardíaca para a compreensão da fisiopatologia da HAS e o cronograma de seleção para novos membros da Liga.

Revista Extensão \& Cidadania, v. 9, n. 16, p. 153-165, jul./dez. 2021. 


\section{Resultados e discussão}

A inscrição para o evento de dissecação de coração foi aberta aos estudantes do primeiro ano do curso de Medicina da UNIFAL-MG ou de outra instituição de ensino superior da cidade. Entretanto, concretizaram a inscrição e compareceram para a realização do evento apenas estudantes da UNIFAL-MG, totalizando 120 estudantes participantes nas duas edições do evento.

Para a realização do evento, cinco extensionistas estavam presentes em cada um dos dias, acompanhando e orientando os participantes. Estes foram distribuídos no laboratório em grupos de cinco pessoas, ocupando um total de quatro bancadas. Em cada uma das bancadas havia um extensionista da Liga para acompanhar os participantes e sanar as dúvidas que pudessem surgir durante a dissecação do coração (Figura 1).

\section{Figura 1 - Laboratório de Anatomia e participantes durante evento promovido pela LADHAS em 2019}

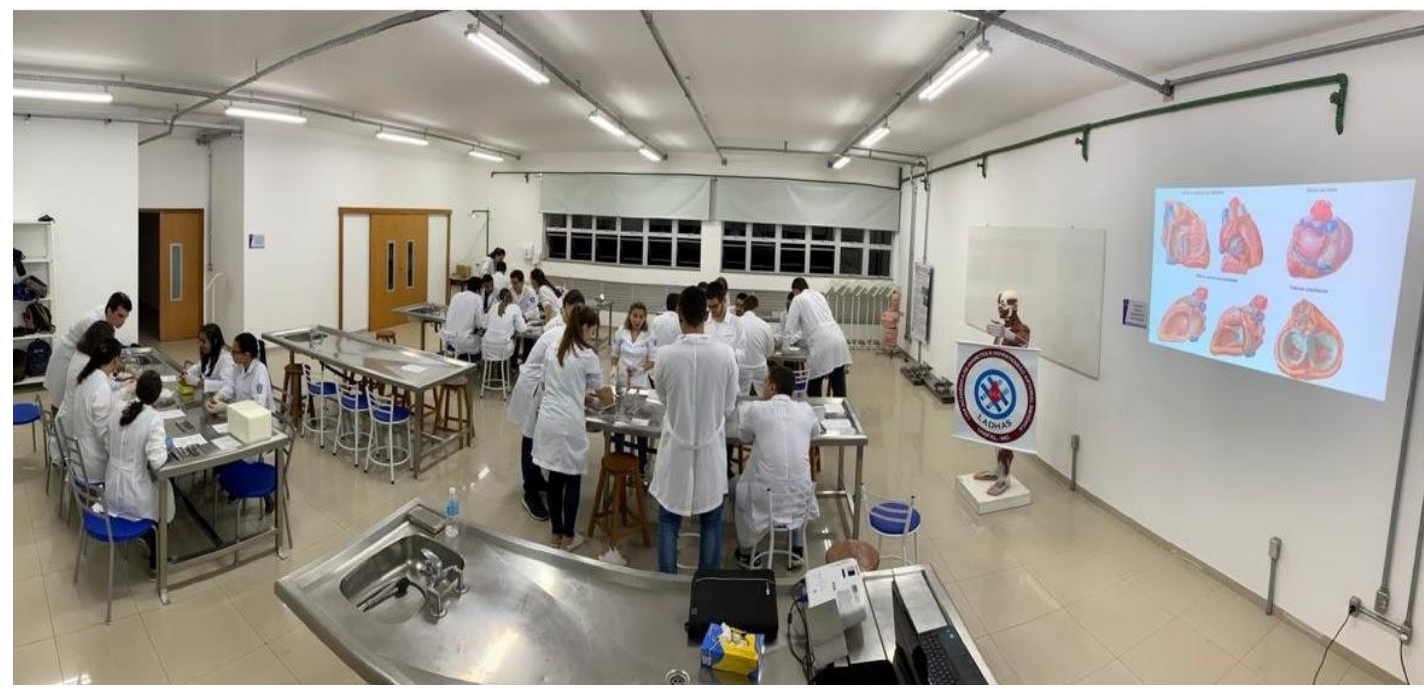

Fonte: Acervo da LADHAS (2019).

Durante a formação acadêmica, nota-se a predominância de metodologias tradicionais no processo de ensino-aprendizagem, nas quais o docente tem a função de transmitir o conteúdo e o discente assume o simples papel de espectador (COSTA et al., 2015). Visando mudar esse modelo de aprendizagem, o evento proporcionou tanto aos extensionistas quanto aos participantes a possibilidade de ocuparem um papel ativo durante a realização da dissecação.

Revista Extensão \& Cidadania, v. 9, n. 16, p. 153-165, jul./dez. 2021. 
Segundo relatos dos extensionistas, o evento ministrado foi de suma importância para o engajamento e enriquecimento da trajetória acadêmica. Nessa perspectiva, foi observado que os integrantes da Liga puderam executar e aperfeiçoar suas habilidades de comunicação, visto que, além de se comunicarem entre si no momento da elaboração do material, tiveram que apresentar o conteúdo a outros estudantes. Dessa forma, no período que antecedeu a realização do evento, os extensionistas se prepararam a fim de aperfeiçoarem suas didáticas e exercerem uma comunicação eficiente na atividade, o que constituiu uma prática bem útil, visto que, no futuro exercício profissional, far-se-á necessária uma interlocução eficaz (RIOS, 2012).

Ademais, outro ponto que pode ser destacado é que essa atividade contribuiu para que os extensionistas aprimorassem a aptidão de liderança e de trabalho em equipe. Esses dois aspectos são fundamentais no contexto dos serviços de saúde, tendo em vista a importância do trabalho em equipe na qualidade do serviço prestado (PEDUZZI; AGRELI, 2018), com destaque para o fato de que o médico pode atuar como líder de equipes nos três níveis de atenção à saúde (primária, secundária e terciária). É válido destacar ainda que, ao proporcionar o trabalho em equipe, baseado na interação e no diálogo, a atividade colabora para que os extensionistas desenvolvam habilidades de comunicação e construam um trabalho colaborativo (BOLLELA et al., 2014).

Outrossim, o evento contribuiu significativamente para a disseminação do saber, uma vez que os extensionistas revisaram um conteúdo já visto anteriormente e tiveram que buscar referências na literatura científica para que pudessem compreender melhor o tema proposto e, dessa forma, ter embasamento técnico para formularem o conteúdo e transmitirem adequadamente o aprendizado para os participantes do evento.

Em relação ao ensino, as atividades desenvolvidas no evento proporcionaram aos participantes o contato com um rico material de estudo anatômico, possibilitando tanto a familiarização com técnicas de dissecação quanto a associação da teoria com a prática. Por permitir a visualização tridimensional das estruturas e o desenvolvimento do raciocínio espacial, a dissecação tem um posto de ferramenta eficiente no aprendizado prático da anatomia. Assim, torna-se evidente que a dissecação contribui para a formação acadêmica dos estudantes - a observação detalhada das peças propicia melhor compreensão da morfologia, enquanto a aplicação de habilidades práticas, a partir da dissecação, leva ao aperfeiçoamento técnico (POCHAT et al., 2011).

Revista Extensão \& Cidadania, v. 9, n. 16, p. 153-165, jul./dez. 2021. 
Cabe destacar ainda que, muitas vezes, o treinamento por meio de peças anatômicas nas universidades é insuficiente para o aprendizado, devido ao mau estado de conservação do acervo, à sua disponibilidade limitada diante do contingente de estudantes e à redução da carga horária de disciplinas básicas do curso de Medicina, entre outros fatores. Portanto, pode existir um déficit a ser suprido no que se refere ao estudo prático da anatomia no meio universitário (NOBESCHI; LOMBARDI; RAIMUNDO, 2018). Nesse contexto, a estratégia adotada no evento promovido pela Liga foi bem-sucedida em seu objetivo de oferecer aos estudantes uma oportunidade de vivenciar a experiência da dissecação a fim de ampliar os conhecimentos acerca da anatomia cardíaca (Figuras 2 e 3).

\section{Figura 2 - Extensionistas da LADHAS apresentando o conteúdo teórico sobre a} anatomia do coração aos participantes do evento
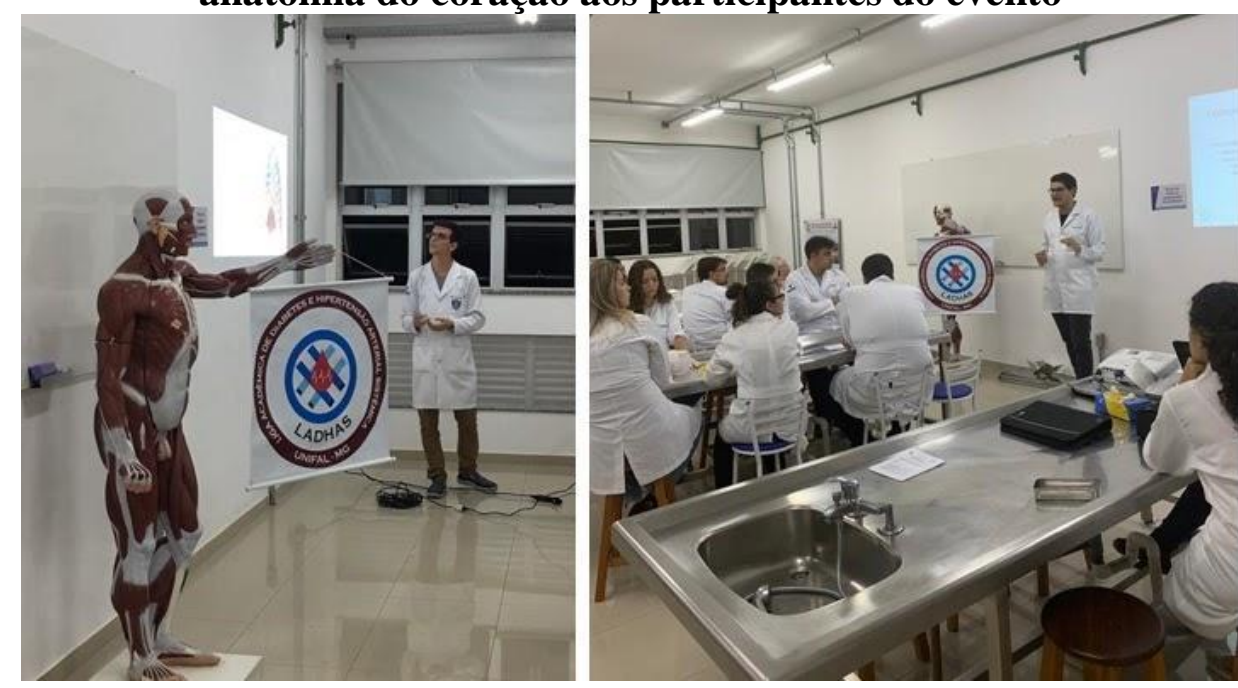

Fonte: Acervo da LADHAS (2019).

Figura 3 - Acadêmica do curso de Medicina realizando a dissecação do coração suíno com supervisão dos extensionistas da LADHAS

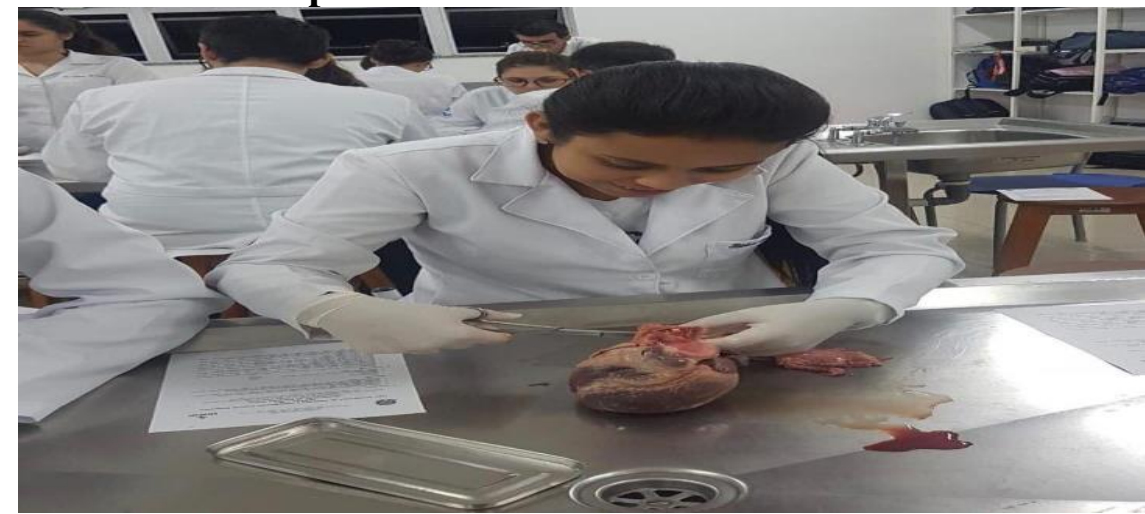

Fonte: Acervo da LADHAS (2019).

Revista Extensão \& Cidadania, v. 9, n. 16, p. 153-165, jul./dez. 2021.

ISSN 2319-0566 DOI: 10.22481/recuesb.v9i16.9110 
Além disso, é válido ressaltar que o conhecimento da anatomia do coração colabora para o entendimento de fisiopatologias cardíacas, como a HAS, promovendo dessa forma a integração básico-clínica. March et al. (2006) destacam que essa integração curricular apresenta vantagens, pois contribui tanto para o aperfeiçoamento do trabalho em equipe quanto para o desenvolvimento e aprimoramento do aprendizado, na medida em que as correlações entre os conteúdos estudados são apresentadas aos estudantes de um modo mais ativo. No caso do curso médico, essa prática faz com que seja favorecido o raciocínio clínico por meio das correlações anatomopatológicas, que preparam os estudantes para as etapas futuras do curso. Além disso, essa integração, atrelada ao compromisso e à ética, estimula o amadurecimento da responsabilidade profissional desde os semestres iniciais do curso.

Considerando ainda que a atividade requer uso de instrumental cirúrgico e cuidados de biossegurança, o evento da liga ofertou aos participantes uma experiência proveitosa por adiantar o contato deles com técnicas e materiais que eles só viriam a conhecer em um momento posterior do curso. Durante a realização do evento, foram ensinados, por exemplo, os nomes das peças do instrumental cirúrgico que foram utilizadas, suas corretas técnicas de manuseio e os cuidados necessários.

Ademais, a atividade foi bastante relevante para mostrar aos estudantes quais as atribuições e atividades realizadas por uma Liga Acadêmica, visto que os participantes eram estudantes dos anos iniciais do curso de Medicina da UNIFAL-MG e haviam ingressado em poucas ou nenhuma das atividades extracurriculares. Desse modo, os participantes puderam conhecer mais sobre a LADHAS, sua atuação perante a comunidade e os trabalhos científicos desenvolvidos por ela, futuramente, podem participar do processo seletivo e vir a ser membros efetivos da Liga.

Os extensionistas e a docente responsável receberam inúmeros elogios sobre a dissecação, os participantes relataram verbalmente e por meio das redes sociais o impacto positivo que a experiência trouxe para eles. Além disso, houve pedidos para que fossem elaborados eventos de dissecação de outros órgãos.

Revista Extensão \& Cidadania, v. 9, n. 16, p. 153-165, jul./dez. 2021. 


\section{Figura 4 - Alguns dos extensionistas da LADHAS no final do evento de dissecação}

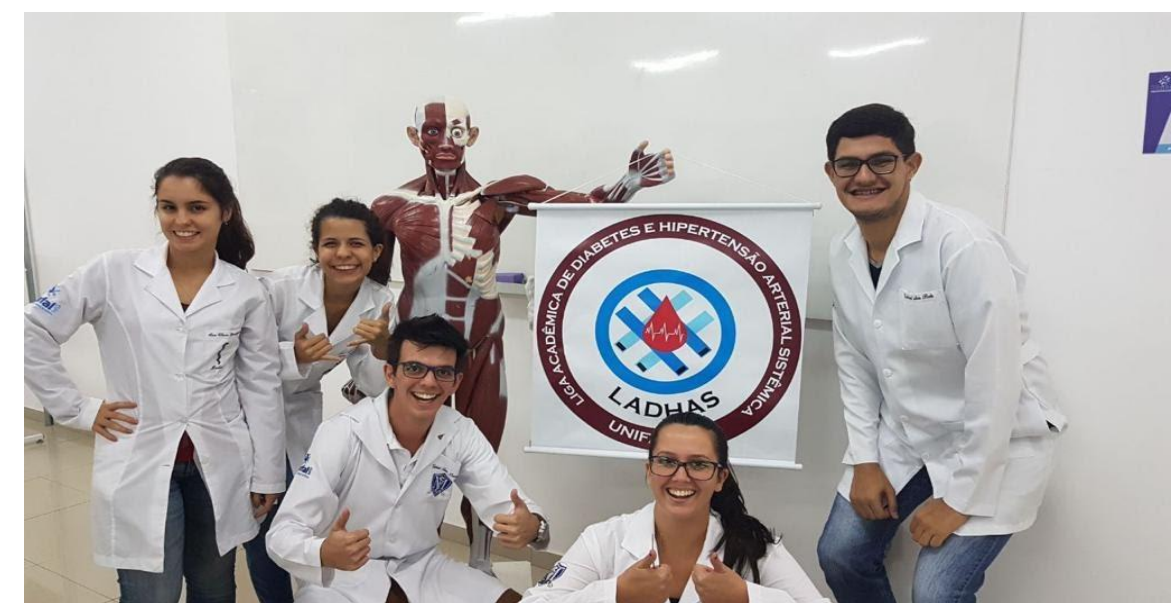

Fonte: Acervo da LADHAS (2019).

Esse feedback positivo incentivou os extensionistas (Figura 4) a investirem nos planos de repetir a atividade todo ano, transformando-a assim em algo tradicional para os acadêmicos do primeiro ano do curso de Medicina. Entretanto, desde o ano de 2020, foi necessária a suspensão das atividades acadêmicas presenciais por conta do cenário da pandemia da Covid19, impossibilitando assim a realização do evento de dissecação.

\section{Considerações finais}

O uso do método ativo nos eventos de dissecação de coração da LADHAS se demonstrou bem atrativo, interessante e facilitador para as futuras associações básico-clínicas que serão apresentadas na formação do curso de Medicina sobre a HAS e sua fisiopatologia. Além disso, por meio do ato de ensinar aos participantes, os extensionistas tiveram a oportunidade de estimular suas habilidades de comunicação. Futuramente, isso poderá auxiliálos em outras ações de educação em saúde, além de facilitar suas inserções no âmbito acadêmico, por meio de monitorias e docência, caso desejem trilhar algum desses caminhos.

Ademais, considerando a essencialidade da anatomia não só para a Medicina, mas também para todos os cursos que envolvem a saúde, a Liga vem estudando planos para aumentar o público-alvo do evento, englobando assim os estudantes das demais áreas da saúde da UNIFAL-MG e de outra instituição de ensino superior de Alfenas. Nesse contexto, a Liga pode contribuir para a democratização do acesso a diversas formas de aprendizado da anatomia no cenário do ensino superior da área da saúde do município.

Revista Extensão \& Cidadania, v. 9, n. 16, p. 153-165, jul./dez. 2021. 
Por fim, tendo em vista os benefícios de aprendizado que o evento trouxe para os participantes em suas edições anteriores, a LADHAS planeja dar continuidade a ele assim que o contexto epidemiológico permitir. Dessa forma, pode-se dar seguimento à intenção de consolidar o evento como atividade tradicional da Faculdade de Medicina da UNIFAL-MG.

\section{Referências}

BARROSO, Weimar Kunz Sebba et al. Diretrizes Brasileiras de Hipertensão Arterial 2020. Arquivos Brasileiros de Cardiologia, v. 116, n. 3, p. 516-658, mar. 2021.

BOLLELA, Valdes Roberto et al. Aprendizagem baseada em equipes: da teoria à prática. Medicina, online, Ribeirão Preto, v. 47, n. 3, p. 293, nov. 2014. Disponível em: https://www.revistas.usp.br/rmrp/article/view/86618/89548. Acesso em: 5 jul. 2021.

COLARES, Maria Alice Mendes et al. Metodologias de ensino de anatomia humana: estratégias para diminuir as dificuldades e proporcionar um melhor processo de ensinoaprendizagem. Arquivos do Museu Dinâmico Interdisciplinar, v. 23, n. 3, p. 140-160, dez. 2019.

COSTA, Raphael Raniere de Oliveira et al. O uso da simulação no contexto da educação e formação em saúde e enfermagem: uma reflexão acadêmica. Espaço para Saúde, Londrina, v. 16, n. 1, p. 59-65, jan./mar. 2015.

LEMOS, Viviane Wosniak. Anatomia comparada do coração de mamíferos domésticos aplicada ao ensino. 2017. Trabalho de Conclusão de Curso (Licenciatura em Ciências Biológicas) - Universidade Tecnológica Federal do Paraná, Dois Vizinhos, 2017.

MALTA, Deborah Carvalho et al. Prevalence of high blood pressure measured in the Brazilian population, National Health Survey, 2013. São Paulo Medical Journal, v. 134, n. 2, p. 163-170, abr. 2016.

MARCH, Cláudia et al. O currículo de Medicina da Universidade Federal Fluminense: revisitando uma experiência. In: PINHEIRO, Roseni Pinheiro; CECCIM, Ricardo Burg; MATTOS, Rubem Araújo (org.). Ensinar Saúde: a integralidade e o SUS nos cursos de graduação na área de saúde. 2. ed. Rio de Janeiro: IMS/UERJ: CEPESC: ABRASCO, 2006. p. 295-309.

MIZERES, Nicholas; GARDNER, Ernest. Métodos de dissecação. Filadélfia: Guanabara Koogan, 1988.

Revista Extensão \& Cidadania, v. 9, n. 16, p. 153-165, jul./dez. 2021. 
NOBESCHI, Leandro; LOMBARDI, Leonardo Augusto; RAIMUNDO, Rodrigo Dominello. Avaliação Sistemática da Dissecação como método de ensino e aprendizagem em Anatomia Humana. Revista Eletrônica Pesquiseduca, v. 10, n. 21, p. 420-432, mai./ago. 2018.

PEDUZZI, Marina; AGRELI, Heloise Fernandes. Trabalho em equipe e prática colaborativa na Atenção Primária à Saúde. Interface - Comunicação, Saúde, Educação, Botucatu, v. 22, n. 2, p. 1525-1534, 2018.

POCHAT, Victor Diniz de et al. Atividades de dissecção de cadáveres e residência médica: relato da experiência do Serviço de Cirurgia Plástica do Hospital Universitário Professor Edgard Santos da Universidade Federal da Bahia. Revista Brasileira de Cirurgia Plástica, v. 26, n. 4, p. 561-565, dez. 2011.

PONTINHA, Carlos Marques; SOEIRO, Cristina. A dissecação como ferramenta pedagógica no ensino da Anatomia em Portugal. Interface - Comunicação, Saúde, Educaçãa, Botucatu, v. 18, n. 48, p. 165-176, 2014.

RIOS, Izabel Cristina. Comunicação em Medicina. Revista de Medicina, São Paulo, v. 91, n. 3, p. 159-162, jul./set. 2012.

RODRIGUES, Hidelgrado. Técnicas anatômicas. 4. ed. Vitória: GM, 2010.

WEBER, John C. Manual de dissecação humana de Shearer. 8. ed. São Paulo: Manole, 2001.

Recebido: 16.07.2021

Aceito: 23.08.2021

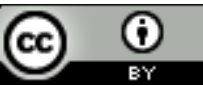

This work is licensed under a Creative Commons Attribution 4.0 International License.

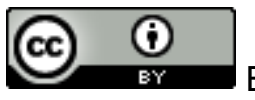

Este trabalho está licenciado com uma Licença Creative Commons - Atribuição 4.0

Revista Extensão \& Cidadania, v. 9, n. 16, p. 153-165, jul./dez. 2021. 